\title{
Determination of radical re-encounter probability distributions from magnetic field effects on reaction yields
}

\author{
Christopher T. Rodgers, ${ }^{\dagger}$ Stuart A. Norman, ${ }^{\dagger}$ Kevin B. Henbest, ${ }^{\dagger \dagger}$ \\ Christiane R. Timmel ${ }^{*,+}$ and P. J. Hore ${ }^{*, \dagger}$
}

${ }^{\dagger}$ Department of Chemistry, University of Oxford, Physical and Theoretical Chemistry Laboratory, South Parks Road, OXFORD, OX1 3QZ, United Kingdom.

Department of Chemistry, University of Oxford, Inorganic Chemistry Laboratory, South Parks Road, OXFORD, OX1 3QR, United Kingdom.

*Authors for correspondence

E-mail: peter.hore@chem.ox.ac.uk, christiane.timmel@chem.ox.ac.uk

\section{Supporting Information}

\section{Regularisation methods}

The central mathematical tools for tackling ill-posed, underdetermined problems are termed "regularisation methods". At the heart of all these methods is the use of some a priori restriction on the set of allowable solutions in order to select a desirable solution from amongst those consistent with the data. Consistency is determined using a suitable statistical test together with an estimate of the noise or uncertainty in the data.

Regularisation allows a balance to be struck between doggedly reproducing every detail of the experimental data-including errors-and producing plausible solutions. Hansen gives a very readable, unified account of many such methods ${ }^{1}$; he also provides an extensive set of Matlab implementations ${ }^{2}$. Using his Matlab toolkit, we have been able to apply a substantial number of regularisation methods to the problem at hand, although we only discuss two in this paper. 


\section{Synthetic data}

Given the difficulty of obtaining reliable solutions to eq (17) and given the plethora of regularisation methods available, we must have some means of evaluating their effectiveness. Here, we used "synthetic data" for this test.

The procedure is as follows:

- We choose some re-encounter probability distribution $f(t)$. In our tests, we used, amongst others, re-encounter probability distributions from the well-known exponential $^{3}$ and diffusion ${ }^{4}$ models of radical pair kinetics.

- We then sample $f(t)$ to give $\mathbf{f}$.

- Using eq (17) in the forward sense, we calculate a set of noiseless synthetic data corresponding to the chosen $f(t)$.

- We then add normally-distributed random noise to the noiseless synthetic data.

- The noisy synthetic data $\mathbf{s}^{\prime}$ are now passed as input to the chosen regularisation method to give a regularised solution $\mathbf{f}_{\mathbf{R}}$.

- The regularised solution $\mathbf{f}_{\mathbf{R}}$ and the original, true re-encounter probability distribution vector $\mathbf{f}$ are compared.

Applying this procedure to a variety of $f(t)$, we are able to chose optimal regularisation parameters which should allow accurate reconstruction of the empirical re-encounter probability $f(t)$ from the experimental data. Significantly, we may avoid the two pitfalls of regularisation methods: under-regularisation, when the solution will show excessive noise; and over-regularisation, when the solution will not bear a strong resemblance to the chosen $f(t)$.

\section{Tikhonov regularisation}

We wish to evaluate critically the ability of Tikhonov regularisation to solve eq (17) and to find the optimum choices of the regularisation matrix $\mathbf{L}$ and the regularisation parameter $\lambda$.

\subsection{Regularisation parameter $\lambda$}

Following conventional practice, we choose an optimal value for $\lambda$ using the L-curve technique ${ }^{5}$. Figure S1 shows an example of an L-curve obtained during Tikhonov regularisation for the hd isotopomer combination using synthetic data created with an exponential model $f(t)$, with added Gaussian noise of standard noise deviation $\sigma_{\text {add }}=0.02$. The L-curve provides a simple visual method of choosing the optimal value of $\lambda$, showing the trade-off between regularisation, which pushes the solution towards one which fits our prior knowledge, and the need to fit the experimental data. On the right hand side, there are three sets of subfigures. In the top pair, we see the case of under-regularisation, where the value of $\lambda$ is too small and the solution is therefore dominated by numerical noise even though the fit to the data is exemplary; this situation corresponds to the top-left end of the L-curve. At the other extreme lies the bottom pair, where $\lambda$ is much too large and the 
solution $f(t)$ is virtually zero everywhere in order to minimise the solution norm, yet the fit to the data is dire; this over-regularised solution corresponds to the bottom-right end of the L-curve. In between these extremes, near the elbow of the L-curve, we strike a balance.

This central region gives the optimal $\lambda$, which fits reasonably well with the synthetic data and which reproduces the true $f(t)$. This is shown in the middle pair of subfigures.
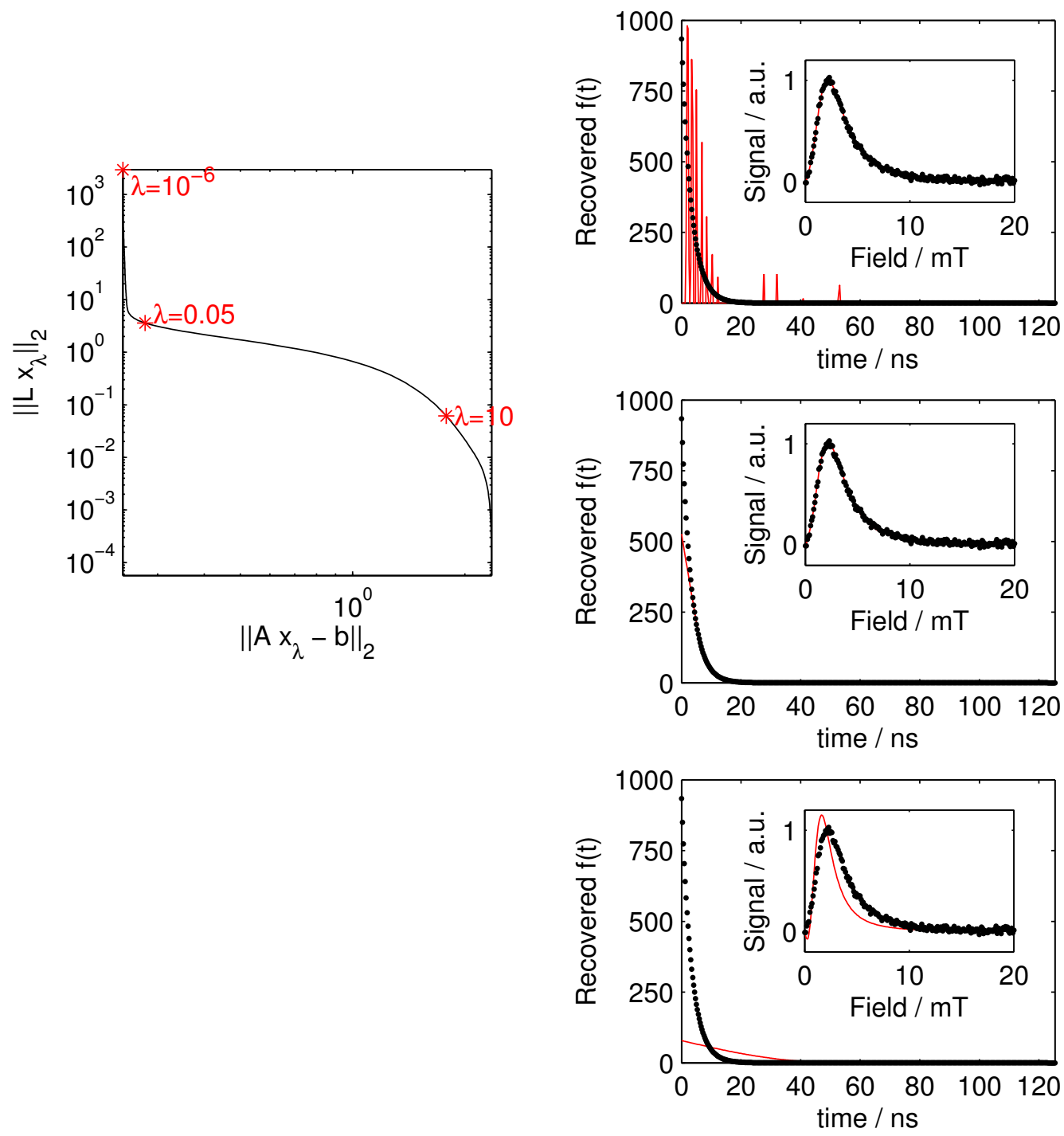

Figure S1. L-curve (left) for exponential model synthetic data in hd. Hyperfine couplings are given in Table 2 of the paper and the rate constant $k=3 \times 10^{8} \mathrm{~s}^{-1}$. The figures on the right show the Tikhonov reconstruction of $f(t)$ in red with the exact $f(t)$ in black. Inset into each figure are the synthetic data in black and the Tikhonov fit in red. Together the figures show underregularisation (top $\lambda=10^{-6}$ ), overregularisation (bottom $\lambda=10$ ) and optimal regularisation (centre $\lambda=0.05$ ). 


\subsection{Regularisation matrix L}

Figure $\mathrm{S} 2$ shows a selection of Tikhonov reconstructions, each with optimal $\lambda$. The figures differ in the choice of solution norm $\mathbf{L}=\mathbf{I}$ (identity matrix) or $\mathbf{L}=\mathbf{D}_{2}$, where $\mathbf{D}_{2}$ is a finitedifferences approximation to the second-derivative operator and in whether $f(t)$ is constrained to be non-negative. Using a solution norm $\mathbf{L}=\mathbf{I}$ will pull the solution $f(t)$ towards zero wherever the data do not provide sufficient evidence for a non-zero value. Physically, this is not a particularly natural choice. Instead, we choose to prefer smooth solutions or, in other words, ones with a small second derivative. We do this by setting $\mathbf{L}=$ $\mathbf{D}_{2}$. Figure $\mathrm{S} 2$ shows that this choice of solution norm produces better reconstructions. The figure also shows the importance of constraining $f(t)$ to be non-negative by using the FNNLS algorithm ${ }^{6}$. 

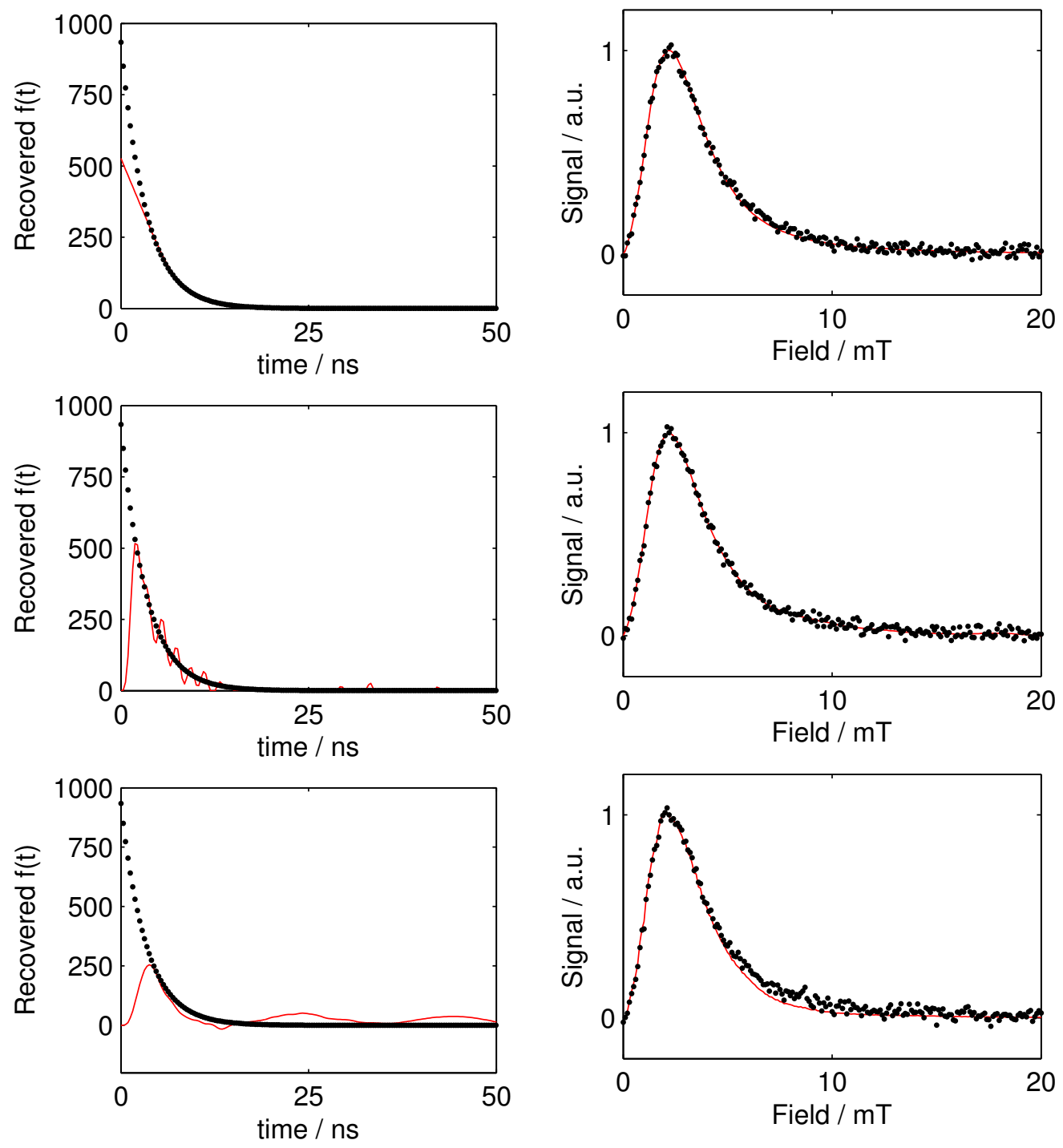

Figure S2. Three sets of Tikhonov reconstructions from synthetic data calculated using an exponential model $\left(k=3 \times 10^{8} \mathrm{~s}^{-1}\right)$ in hd. The hyperfine coupling data were taken from Table 2. The L-curve method was used to choose an optimal value of $\lambda$ in each plot. The recovered $f(t)$ were essentially zero outside the range shown. Top: $L=D_{2}$ and using FNNLS ${ }^{6}$. Middle: $L=I$ and using FNNLS. Bottom: $L=I$ and using Hansen's SVD-based Tikhonov method ${ }^{2}$. Notice that the best reconstruction arises when we enforce nonnegative values for $f(t)$ and when we use $L=D_{2}$ which penalises oscillatory solutions.

\subsection{Evaluation}

Although not shown here, Tikhonov regularisation methods were also tested against other model re-encounter probabilities $f(t)$ such as the diffusion model ${ }^{4}$, or a single Gaussian peak centred at a chosen time. In each case, choosing $\mathbf{L}$ to approximate the second derivative operator was found to give the best reconstruction. Together, these results suggest that Tikhonov regularisation is capable of recovering the re-encounter probability $f(t)$ from the 
experimental data when $\mathbf{L}=\mathbf{D}_{2}$ and when the FNNLS algorithm is used to force a nonnegative solution.

\section{Maximum entropy regularisation}

When using the maximum entropy method for regularisation, we must also choose two parameters: the baseline parameter $b$ and an estimate of the noise $\sigma$ present in the recorded signal. We use synthetic data to investigate the most appropriate choices of these parameters for recovering $f(t)$.

\subsection{Baseline parameter}

Figure S3 shows a series of maximum entropy reconstructions with different values of the baseline parameter $b$. We see that the overall shape of the re-encounter probability $f(t)$ is recovered for a wide range of $b$ values. However, we may also note that increasing the value of the baseline parameter $b$ decreases the tendency of the maximum entropy method to produce sharp peaks. Whilst sharp peaks are desirable when reconstructing overlapping spectral features - for example, in NMR spectra - they do not make good physical sense in this context and therefore, we will tend to choose larger values of $b$ than have typically been used to process NMR data ${ }^{7,8}$.
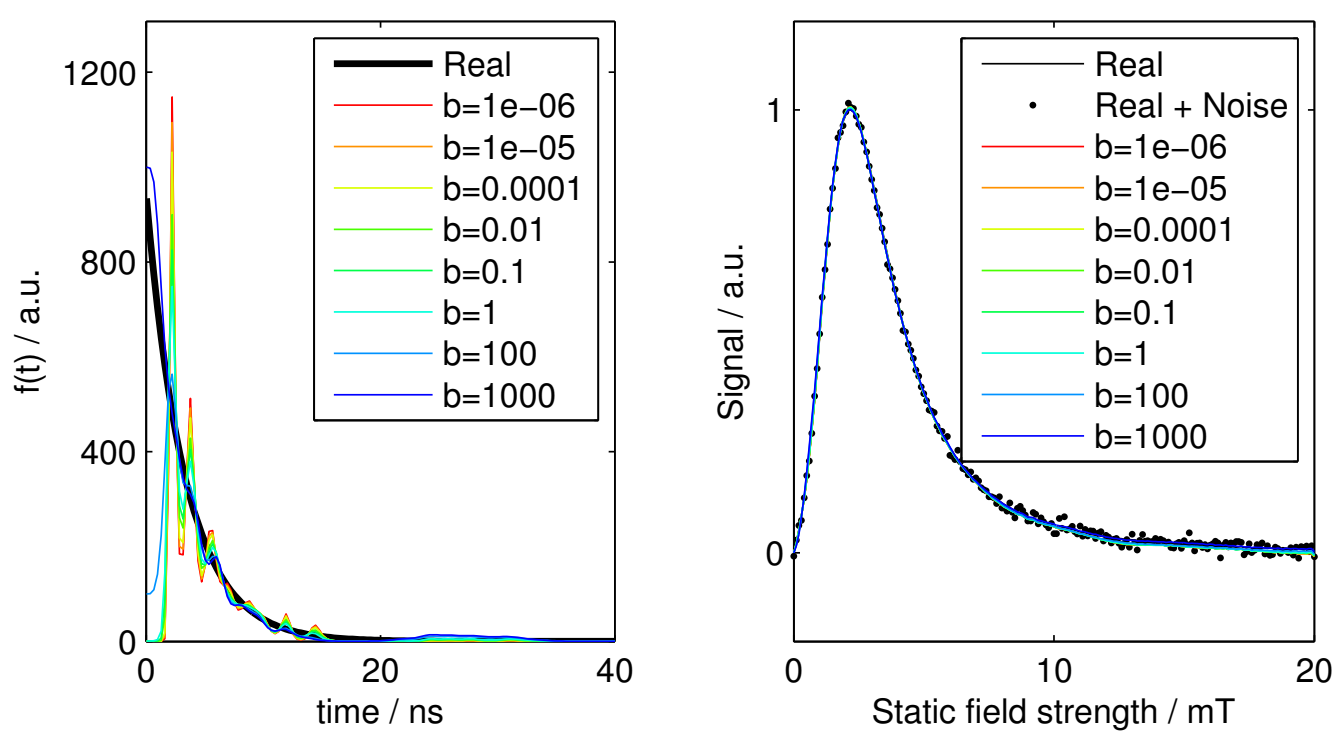

Figure S3. Maximum entropy reconstructions of $f(t)$ in the hd isotopomer (hyperfine data were taken from Table 2). Synthetic data were generated using the exponential model $\left(k=3 \times 10^{8} \mathrm{~s}^{-1}\right)$ shown in black with normally-distributed random noise added $\left(\sigma_{\text {add }}=0.01\right)$. The maximum entropy noise estimate $\sigma=0.01$. The different reconstructions show how the parameter $b$ influences $f(0)$ and also the oscillations in the recovered $f(t)$. Notice that the fit to the experimental data (right) is exemplary in each case. This illustrates the ill-posed nature of this problem; many $f(t)$ are consistent with the data. 


\subsection{Noise estimate}

Figure S4 shows another series of maximum entropy reconstructions from the same system, where we set $b=100$ and explore the effects of altering the noise estimate $\sigma$. Clearly, the quality of the recovered $f(t)$ improves as the noise estimate approaches the true noise level $\sigma_{\text {add }}$. Notably, $f(t)$ is recovered properly at earlier times as $\sigma$ is reduced. On the other hand, attempts to use a noise estimate $\sigma$ smaller than the true noise level $\left(\sigma_{\mathrm{add}}=0.01\right)$ resulted in the method failing to converge. In light of these results, we adopt a pragmatic approach when analysing the experimental data and choose the lowest value of $\sigma$ at which the maximum entropy solution converges within 1000 iterations.

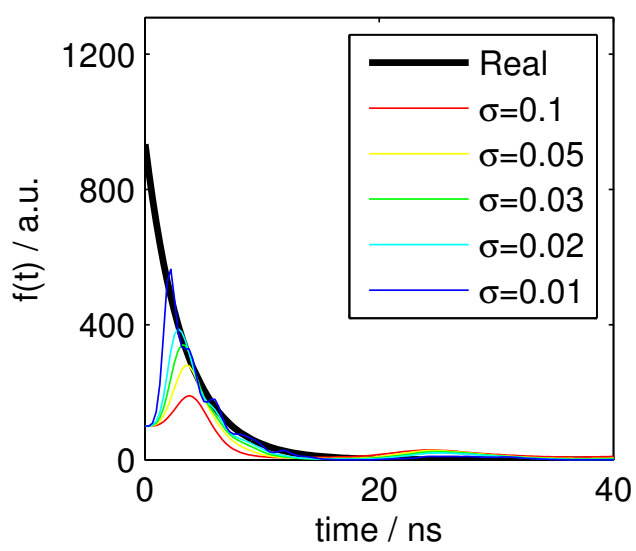

Figure S4. Maximum entropy reconstructions of $f(t)$ in the hd isotopomer (hyperfine data were taken from Table 2). Synthetic data were generated using the exponential model $\left(k=3 \times 10^{8} \mathrm{~s}^{-1}\right)$ shown in black with normally-distributed random noise added $\left(\sigma_{\text {add }}=0.01\right)$. The maximum entropy parameter $b=100$ in all cases. The different reconstructions show how the estimated noise standard deviation $\sigma$ influences the recovered $f(t)$. The maximum entropy method was found not to converge for $\sigma<0.01$ (i.e. when $\sigma<\sigma_{\text {add }}$ ).

Figure S5 summarises the results of a large series of maximum entropy calculations on this same set of synthetic data. For each converged solution, we plot a point whose coordinates indicate the errors in fitting the synthetic data and the errors in recovering the true $f(t)$. This figure supports the argument above that a value of $b \sim 100-1000$ and the smallest possible noise estimate $\sigma$ are close to being optimal. Other analogous calculations were performed for synthetic data from the diffusion model and for a re-encounter probability having the form of a single Gaussian peak. The results in these cases support the same conclusion. 


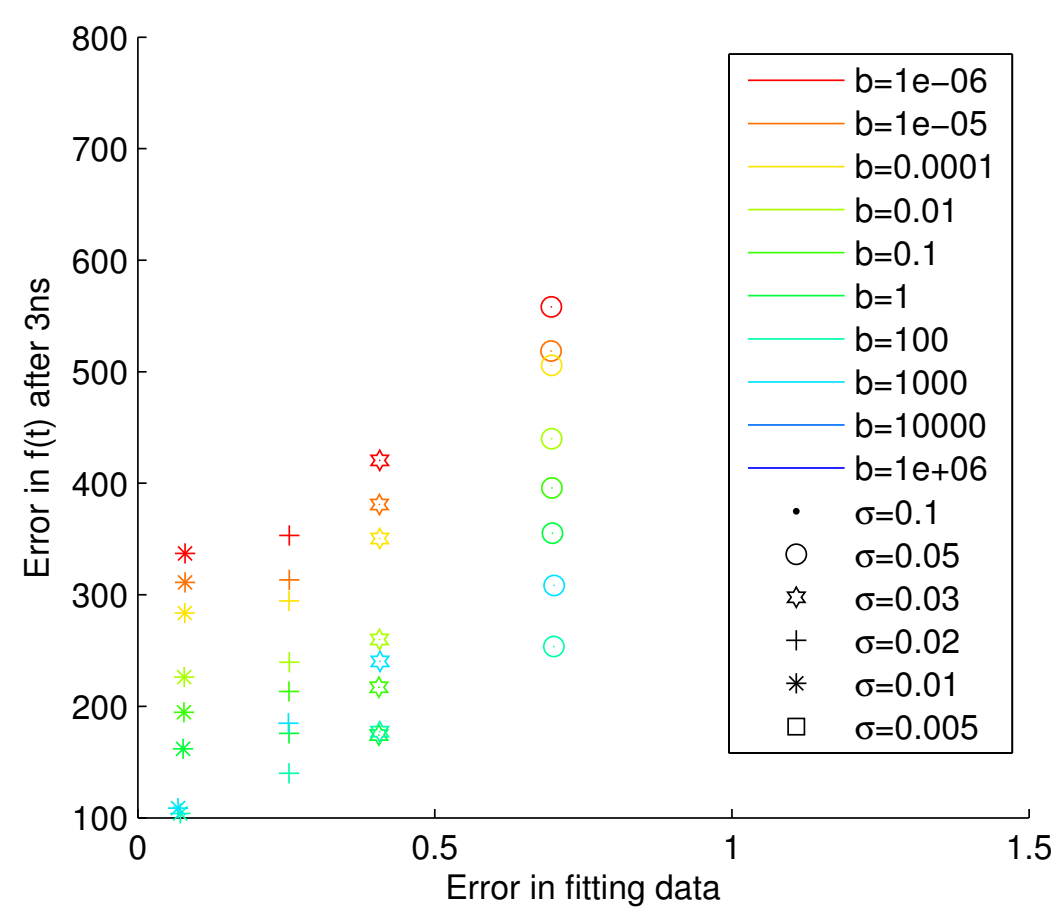

Figure S5. Summary of several maximum entropy reconstructions of $f(t)$ in the hd isotopomer. Synthetic data were generated using an exponential model $f(t)$ with normally-distributed random noise added $\left(\sigma_{\text {add }}=0.01\right)$. Reconstructions were attempted with a variety of $b$ and $\sigma$ values. For each converged reconstruction, we plot a point showing the residual norm in recovering $f(t)$ vs the norm of the deviations from the synthetic data. Notice that the best results are when $\sigma$ matches the true noise standard deviation and with a reasonably large value of $b$. Underestimating $\sigma$ or choosing an excessive $b$ causes maximum entropy not to converge. Overestimating $\sigma$ or using a small $b$ gives rise to a suboptimal reconstruction.

\subsection{Evaluation}

Maximum entropy regularisation works reasonably well with synthetic data for the hd isotopomer combination. The two most significant difficulties are a failure to reconstruct $f(t)$ for times shorter than $\sim 2 \mathrm{~ns}$ and a tendency to produce peaked, oscillatory solutions, as discussed in the main text. The failure to recover $f(t)$ shortly after creation is thought to arise because spin evolution in the radical pair depends very little on the magnetic field strength in these first few nanoseconds. As discussed in the main text, this means that the first derivative experimental data do not constrain $f(t)$ in these first instants, explaining the observed discrepancies. At later times, the spin evolution depends more strongly on the magnetic field strength and this difficulty no longer arises.

We illustrate this reasoning by calculating the singlet probability in the hd isotopomer for 201 magnetic fields between 0 and $20 \mathrm{mT}$ and at many times during the first $50 \mathrm{~ns}$. From these data, we calculate the standard deviation of the singlet probabilities at different field strengths for a given time. This standard deviation is shown in Figure S6, giving a 
qualitative measure of the extent to which the singlet probability depends on magnetic field strength in the hd isotopomer during the first 50 ns. Hence, Figure S6 also gives a qualitative measure of the extent to which $f(t)$ is constrained by the experimental data during this period.

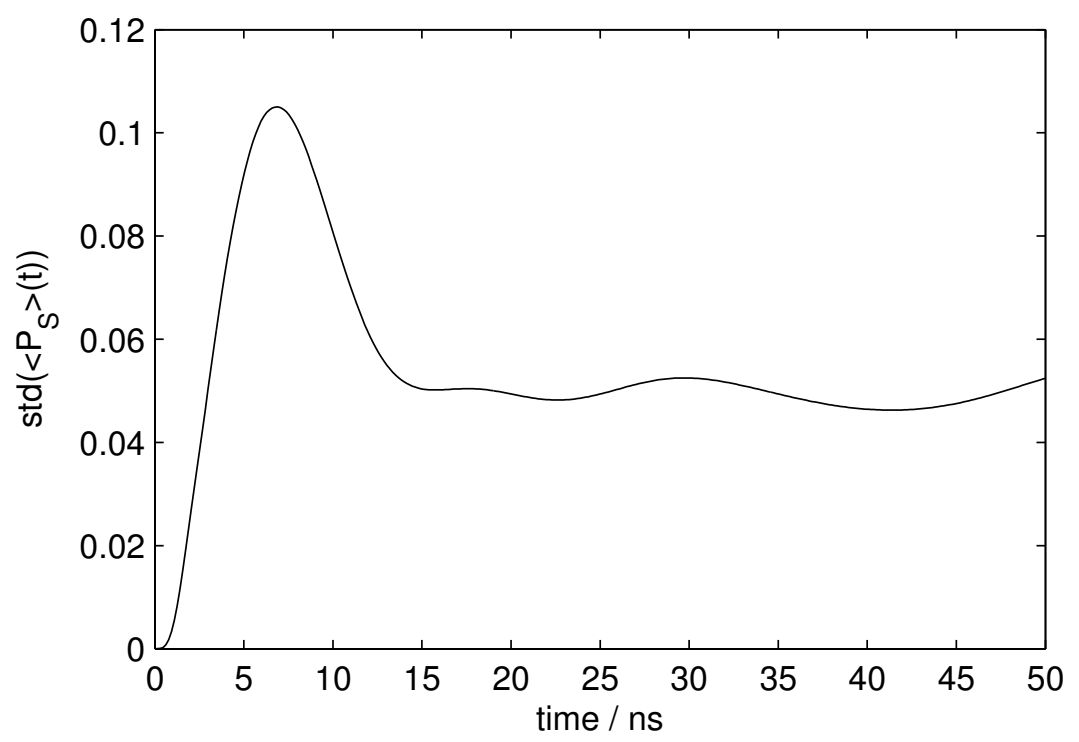

Figure S6. Standard deviation of the singlet probability in hd at each of the 201 field points plotted as a function of time. Notice that in the first few nanoseconds after radical pair creation, there is very little change in spin evolution at different static field strengths. This means that the experiment is insensitive to $f(t)$ at short times. The standard deviation remains roughly constant around 0.05 at times greater than $15 \mathrm{~ns}$. 


\section{Examples of MaxEnt and Tikhonov fits}

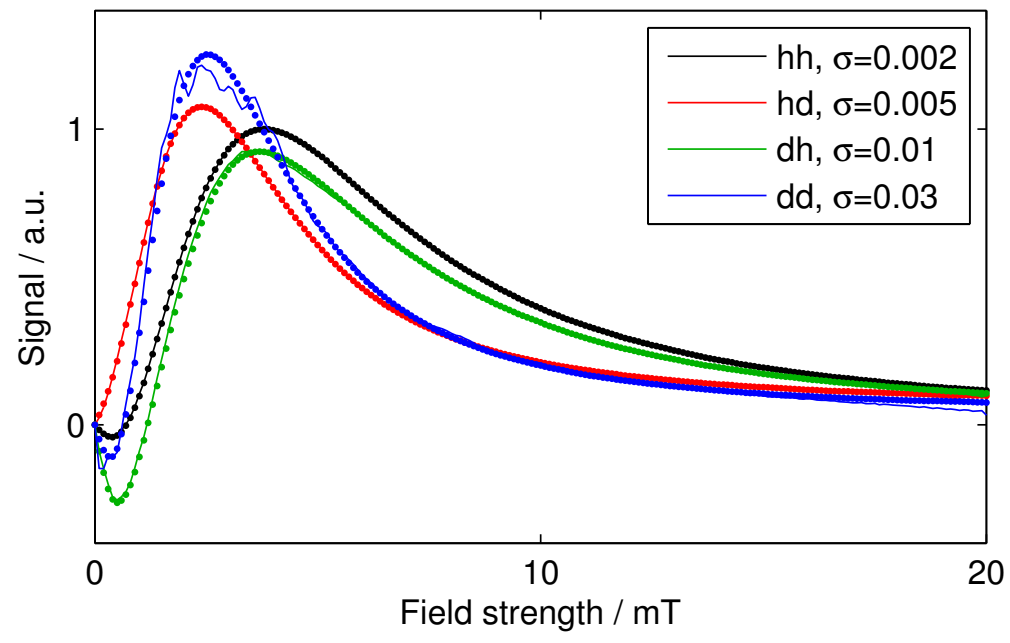

Figure S7. Best fits to the experimental data using maximum entropy regularisation. The maximum entropy noise estimates $\sigma$ for each isotopomer combination are given in the legend. These were chosen to be as small as possible, as described in the main text. Notice that the fits are exemplary, except for a small region in the dd isotopomer around $3 \mathrm{mT}$ which is discussed in the main text. This figure complements Figure 2B in the main text.

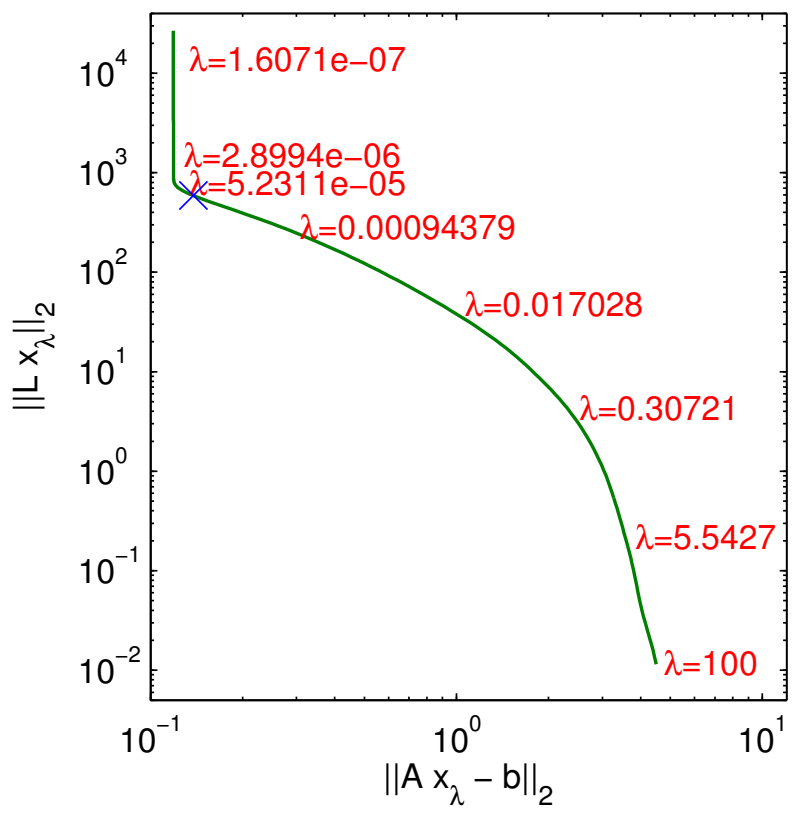

Figure S8. Illustrative L-curve showing the trade off between solution and residual norm for Tikhonov regularisation in the $\mathbf{d h}$ isotopomer pair with different choices of the regularisation parameter $\lambda$. The optimal value of $\lambda$, indicated with a blue cross, is just below and to the right of the "L". This L-curve corresponds to the green curve in Figure 2A from the main text. 


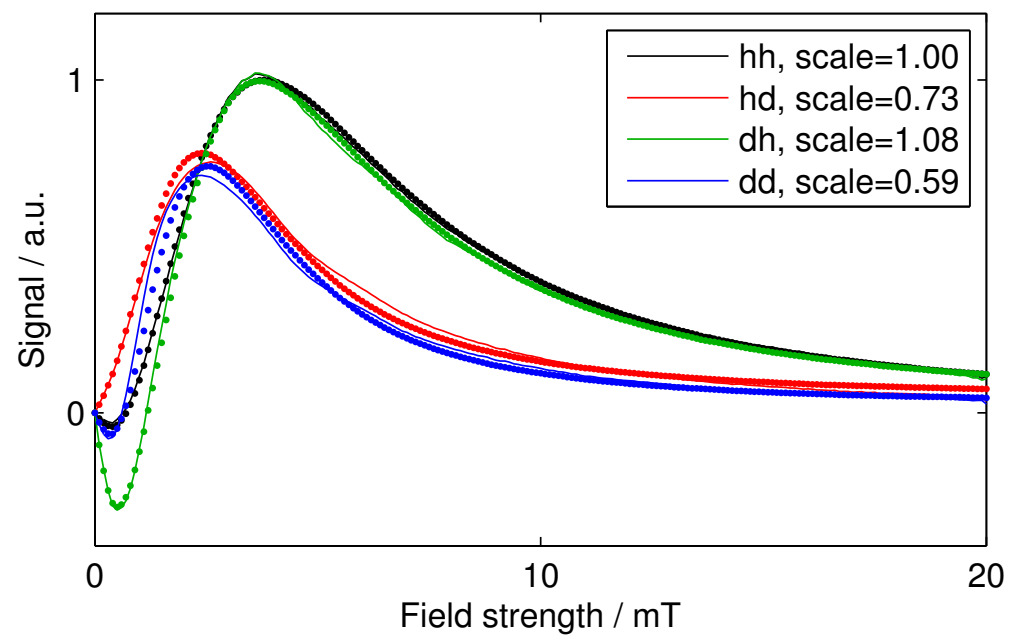

Figure S9. Best fits to the experimental data using Tikhonov regularisation applied to all four data sets simultaneously in order to produce a single re-encounter probability $f(t)$. The regularisation parameter $\lambda=0.0002$ was chosen using the L-curve method. The experimental data were multiplied by optimised values of "scale" before using Tikhonov regularisation. Notice that the fit is exemplary, except for small deviations in the dd isotopomer curve. This figure complements Figure 2C from the main text.

\section{Quantum mechanical Monte Carlo (QMMC) method}

In an attempt to shed light on the dependence of the LFE depth and $B_{1 / 2}$ on the values of the hyperfine coupling constants in a multinuclear radical pair, we have calculated the magnetic field responses - which we here call Magnetically Affected Reaction Yield (MARY) spectra - for an ensemble of radical pairs, in the frequency domain using eq (11) without binning. The hyperfine coupling constants are chosen at random for any particular radical pair. The calculated MARY spectra are then analysed to obtain the LFE depth and $B_{1 / 2}$. Across the whole ensemble, these parameters will take on values according to some probability distribution, providing that enough MARY spectra have been calculated to minimise fluctuations. Then, the problem becomes a matter of analysing the probability distributions, often graphically, to determine the most important, controlling combination of variables for each empirical parameter. For example, we may pose questions such as: does the low field effect depth vary with the ratio of the effective hyperfine couplings on each radical? Such a quantum mechanical Monte Carlo (QMMC) approach will not explain why a parameter is controlled by particular variables, but neither does it need such knowledge (in the form of physical assumptions) to be provided in advance.

MARY spectra were calculated in an ensemble of 12,737 radical pairs. Each member of the ensemble had six random hyperfine couplings to spin- $-1 / 2$ nuclei-three on each radical. These hyperfine couplings were chosen such that the effective HFCs on each radical were uniformly distributed between 0 and $1.5 \mathrm{mT}$. After selection of the HFCs, MARY spectra were calculated for each member of the ensemble at 1000 field strengths varying 
logarithmically between $10^{-11}$ and $10 \mathrm{mT}$ and also at zero field. Exponential model rate constants were taken between $10^{-2}$ and $10^{12} \mathrm{~s}^{-1}$, which covers the typical experimental values and several orders of magnitude on either side. In total, the QMMC calculation involved more than $3 \times 10^{8}$ singlet yield evaluations.

\subsection{The LFE depth}

Figure S10 shows the variation of the LFE depth extracted from the QMMC MARY spectra with the effective hyperfine coupling constants of the two radicals, for three different exponential model rate constants. We plot both the mean LFE depths for members of the ensemble having effective HFCs within each rectangle and also their standard deviations. In the limit of slow recombination kinetics, when $k<<\left\langle a_{\mathrm{A}, \mathrm{B}}\right\rangle / \gamma_{\mathrm{e}} \approx 10^{8} \mathrm{~s}^{-1}$, the LFE depth is virtually independent of the hyperfine couplings (Figures $S 10(a)$ and $S 10(b)$ ) as reported by Timmel et al. ${ }^{9}$ For intermediate kinetics, when $k \approx\left\langle a_{\mathrm{A}, \mathrm{B}}\right\rangle / \gamma_{\mathrm{e}} \approx 10^{8} \mathrm{~s}^{-1}$, the LFE depth is rather small unless $\left.\left\langle a_{\mathrm{A}}\right\rangle>\right\rangle\left\langle a_{\mathrm{B}}\right\rangle$ or vice-versa (Figures $\mathrm{S} 10(\mathrm{c})-\mathrm{S} 10(\mathrm{f})$ ). However, when $\left\langle a_{\mathrm{A}}\right\rangle$ $>>\left\langle a_{\mathrm{B}}\right\rangle$ we also find the largest standard deviation in the LFE depth. Hence, for rate constants comparable to the effective HFCs, radical pairs with dominant HFCs on one partner may, but need not necessarily, show a deep LFE. Finally, for rapid kinetics, when $k$ $>>\left\langle a_{\mathrm{A}, \mathrm{B}}\right\rangle / \gamma_{\mathrm{e}} \approx 10^{8} \mathrm{~s}^{-1}$, a LFE is not seen and the field effect curve increases monotonically to the $\Phi_{\mathrm{S}}(\infty)$ value. 

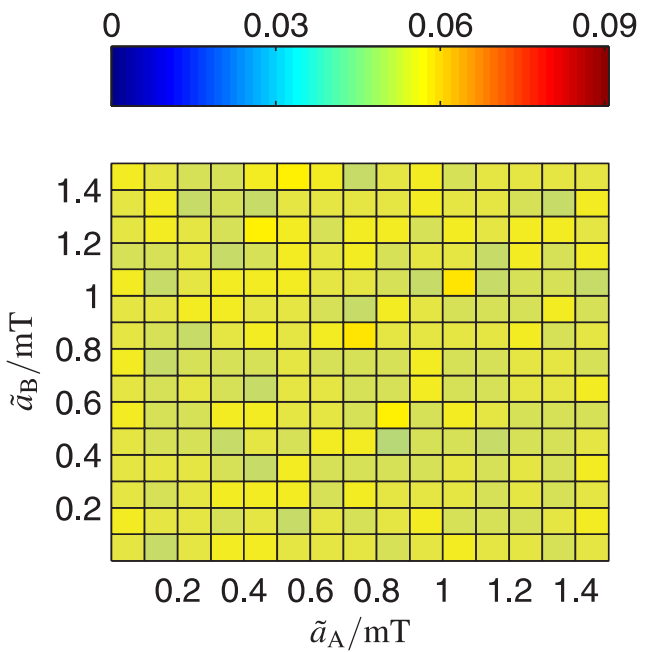

(a) Mean LFE depth when $k=0.01 \mathrm{~s}^{-1}$.

Values in range 0.05-0.06.

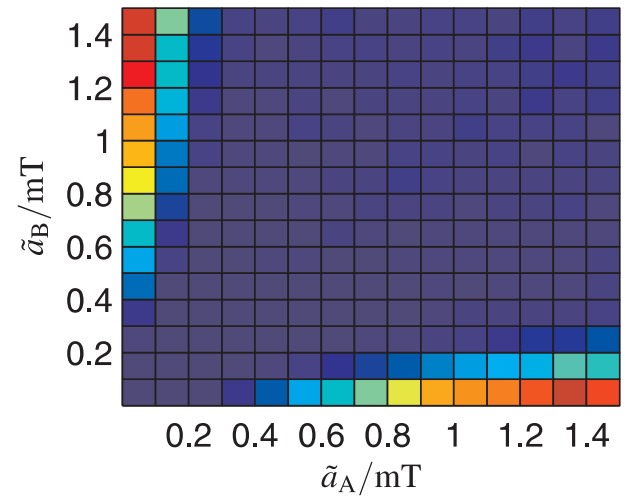

(c) Mean LFE depth when $k=3 \times 10^{7} \mathrm{~s}^{-1}$. Values in range $0.00-0.08$.

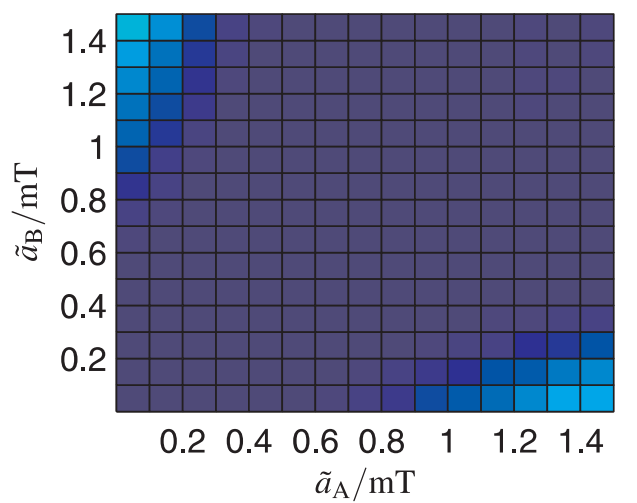

(e) Mean LFE depth when $k=8 \times 10^{7} \mathrm{~s}^{-1}$. Values in range 0.00-0.04.
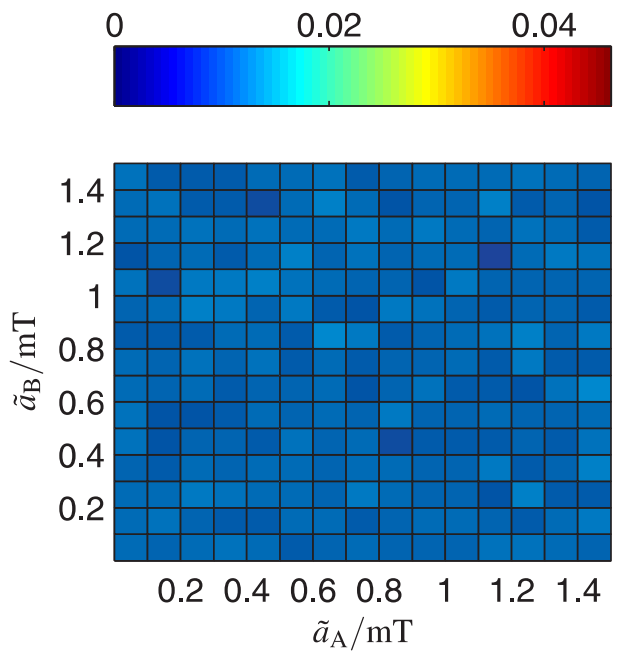

(b) Standard deviation in LFE depth when $k=0.01 \mathrm{~s}^{-1}$. Values in range $0.01-0.01$.

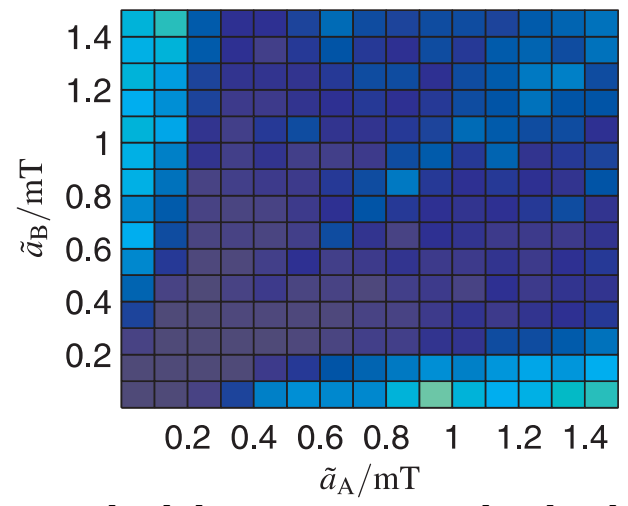

(d) Standard deviation in LFE depth when $k=3 \times 10^{7} \mathrm{~s}^{-1}$. Values in range 0.00-0.02.

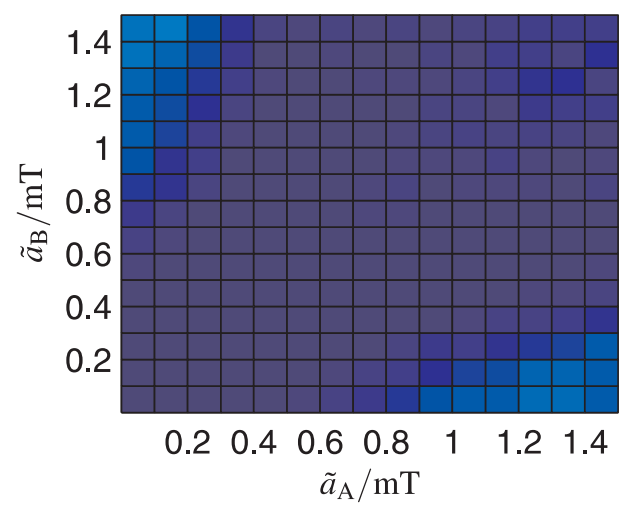

(f) Standard deviation in LFE depth when $k=8 \times 10^{7} \mathrm{~s}^{-1}$. Values in range 0.00-0.01.

Figure S10: LFE depth according to QMMC calculations in an ensemble of 12,737 radical pairs. The colour of each rectangle summarises the results from those members of the ensemble having effective HFCs which fall within it. We plot the mean LFE depth in each cell on the left and the standard deviations on the right. Rate constants $k$ were selected to be less than and comparable to the effective HFCs. Calculations with $k>5 \times$ $10^{8} \mathrm{~s}^{-1}$ showed no LFE at all and are therefore not presented. 


\subsection{The half-saturation field, $B_{1 / 2}$}

Figure S11 gives a further analysis of the MARY spectra from the QMMC calculation above. In each radical pair, the saturation singlet yield $\Phi_{\mathrm{S}}(\infty)$ was calculated using the high field limit (i.e. the secular approximation). This gave the singlet yield corresponding to $B_{1 / 2}$ : $\Phi_{\mathrm{S}}\left(B_{1 / 2}\right)=1 / 2\left[\Phi_{\mathrm{S}}(0)+\Phi_{\mathrm{S}}(\infty)\right]$. Thence, $B_{1 / 2}$ was determined for each member of the ensemble by interpolation of the calculated MARY spectrum. We may now examine the factors governing $B_{1 / 2}$.

Figures S11(a) and S11(b) show the mean and standard deviation of $B_{1 / 2}$ as a function of effective HFC. It is clear that the situation where the effective HFC on one radical dominates (e.g. $\left\langle a_{\mathrm{A}}\right\rangle>>\left\langle a_{\mathrm{B}}\right\rangle$ ) allows - but does not require - a large value for $B_{1 / 2}$.

In order to test further the reliability of Weller's empirical formula, eq (2), Figures S11(c)S11(f) show scatter plots of the actual calculated $B_{1 / 2}$ value versus the $B_{1 / 2}$ value predicted using Weller's formula. Each point in the plots represents one member of the QMMC ensemble of radical pairs. It is immediately obvious that there is frequently a sizeable scatter in the figures.

When the rate constant is smaller than the effective HFCs (Figure S11(c)), we see that the Weller formula provides something of an upper bound for the true $B_{1 / 2}$. The Weller formula may overestimate $B_{1 / 2}$ by around a factor of two.

When the rate constant is similar to the effective HFCs (Figures S11(d) and S11(e)), we see that the Weller formula and the calculated $B_{1 / 2}$ values correlate reasonably well. The Weller formula clearly captures the average behaviour of the exact $B_{1 / 2}$ values. Nevertheless, there is a scatter of $\pm 30 \%$, and in some cases the exact $B_{1 / 2}$ value significantly exceeded Weller's predictions. At intermediate rate constants, the Weller formula is thus only a useful rule of thumb for predicting the $B_{1 / 2}$ value.

Finally, when the rate constant is significantly faster than the effective HFCs, the Weller formula gives a dramatic underestimate of the $B_{1 / 2}$ value. 


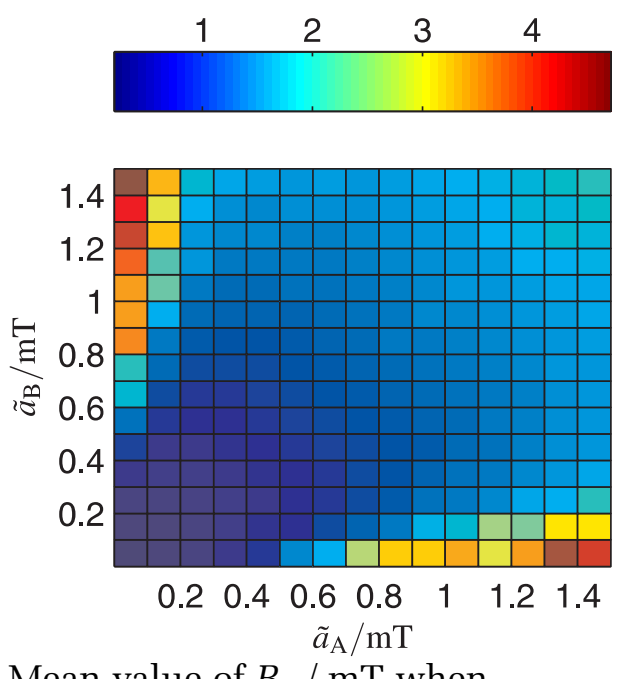

(a) Mean value of $B_{1 / 2} / \mathrm{mT}$ when $k=3 \times 10^{7} \mathrm{~s}^{-1}$

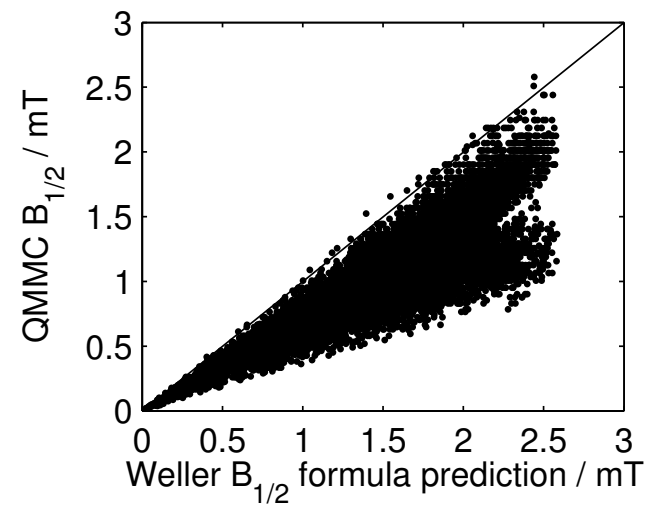

(c) QMMC $B_{1 / 2}$ vs. eq (2) when $k=0.01 \mathrm{~s}^{-1}$.

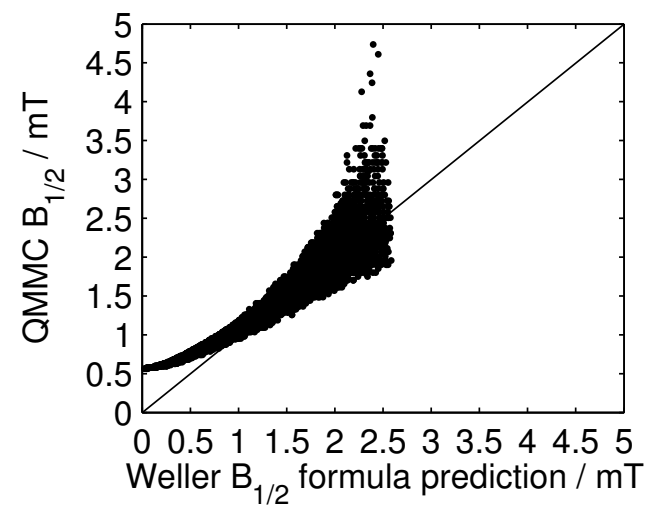

(e) QMMC $B_{1 / 2}$ vs. eq (2) when $k=10^{8} \mathrm{~s}^{-1}$.

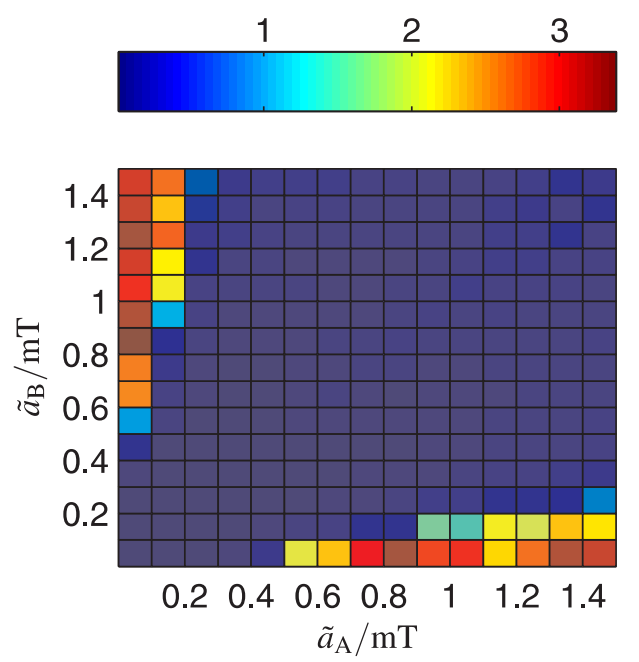

(b) Standard deviation in $B_{1 / 2} / \mathrm{mT}$ when $k=3 \times 10^{7} \mathrm{~s}^{-1}$.

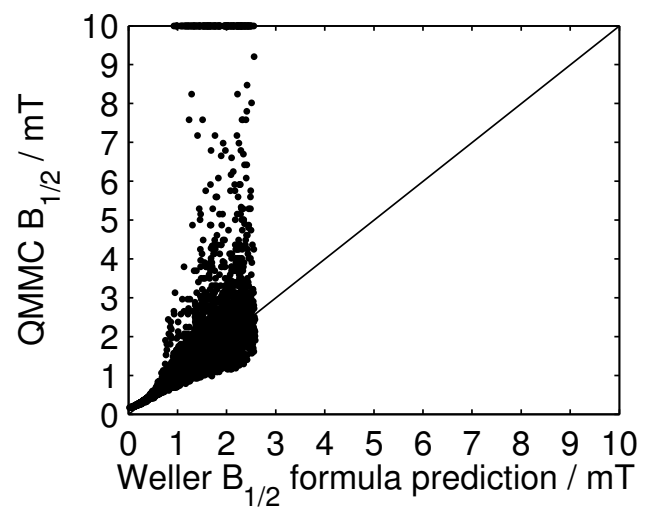

(d) QMMC $B_{1 / 2}$ Vs. eq (2) when $k=3 \times 10^{7} \mathrm{~s}^{-1}$.

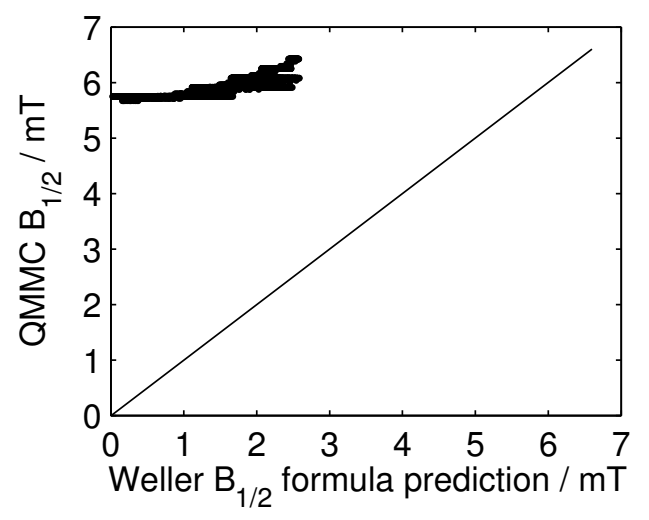

(f) QMMC $B_{1 / 2}$ vs. eq (2) when $k=10^{11} \mathrm{~s}^{-1}$.

Figure S11: Half-saturation field $B_{1 / 2}$ according to QMMC calculations in 12,737 radical pairs. (a-b): The colour of each rectangle summarises the results from those members of the ensemble having effective HFCs which fall within it. We plot the mean $B_{1 / 2} / \mathrm{mT}$ in each cell on the left and the standard deviations on the right. (c-f): Each point represents one radical pair from the ensemble. Were the Weller formula-given in eq (2)-perfect, all points would lie on the diagonal line. Rate constants were chosen to be smaller than (c), comparable to (a-b, d-e) and larger than (f) the effective HFCs. 


\section{The use of eq (3) to calculate the magnetic field effect on the reaction product yield - a validation}

There is a possibility that by separating the spin evolution of the radical pair from its diffusive motion, as we do by using eq (3), some important contribution to the magnetic field effect on the singlet yield, $\Phi_{\mathrm{S}}$ might be missed. To address this point, we compare the magnetic field dependence of $\Phi_{\mathrm{S}}$ (for a one-proton radical pair) predicted using the approximate method described in the article with that obtained from an exact numerical solution of the stochastic Liouville equation (SLE) using the method of Pedersen and Freed. ${ }^{10}$ The conditions of the simulations were: hyperfine coupling constant $=1.0 \mathrm{mT}$; diffusion coefficient $D=10^{-9}, 10^{-10}$, or $10^{-11} \mathrm{~m}^{2} \mathrm{~s}^{-1}$; initial separation $=1.0 \mathrm{~nm}$; encounter separation $=0.5 \mathrm{~nm}$; instantaneous reaction of singlet states at the encounter separation; and an exponential exchange interaction:

$J(R)=J_{0} \exp (-R / \alpha)$

with either $J_{0}=0$, or with $J_{0}=7.1 \times 10^{5} \mathrm{~T}, \alpha=0.0467 \mathrm{~nm}$ (typical values for radical pairs in solution, taken from a study of acyl-ketyl biradicals by Tsentalovich et al. ${ }^{11}$ see also O'Dea et al. ${ }^{12}$ )

As may be seen in Figure S12, in the absence of an exchange interaction between the radicals, the shape of the magnetic field effect calculated using the approximate approach is essentially identical to the SLE results for all three values of the diffusion coefficient, except for a scaling factor $a$ and a constant offset $b$, both of which are independent of the magnetic field strength, $B$. That is, the two singlet yields are related by:

$\Phi_{\mathrm{S}}(B)^{\mathrm{SLE}}=a \Phi_{\mathrm{S}}(B)^{\mathrm{approx}}+b$

Our experiments contain no information on the values of either $a$ or $b$, because we measure the first derivative of the fluorescence intensity and have no way of determining the absolute size of the change in $\Phi_{\mathrm{s}}$. When a physically reasonable exchange interaction is introduced, via the parameters given above, the scaling and offset (i.e. $a$ and $b$ ) of the magnetic field dependence of $\Phi_{\mathrm{S}}$ changes, as expected, ${ }^{12}$ but its shape remains essentially unchanged for $D=10^{-9}$ and $10^{-10} \mathrm{~m}^{2} \mathrm{~s}^{-1}$ and changes only minimally for the smallest diffusion coefficient, $D=10^{-11} \mathrm{~m}^{2} \mathrm{~s}^{-1}$. We estimate a diffusion coefficient of $\sim 10^{-9} \mathrm{~m}^{2} \mathrm{~s}^{-1}$ under the conditions of our experiments $\left(T=298 \mathrm{~K}, \eta=5 \times 10^{-4} \mathrm{~kg} \mathrm{~m}^{-1} \mathrm{~s}^{-1}, r=0.5 \mathrm{~nm}\right)$.

To summarize, the errors introduced by calculating the singlet yield in the way we have proposed, with the spin evolution separated from the diffusive motion, and without considering exchange explicitly, amount simply to a scaling and an offset in $\Phi_{\mathrm{S}}$, neither of which can be detected by our experiments. We are therefore confident that our approach is valid. 
(A)
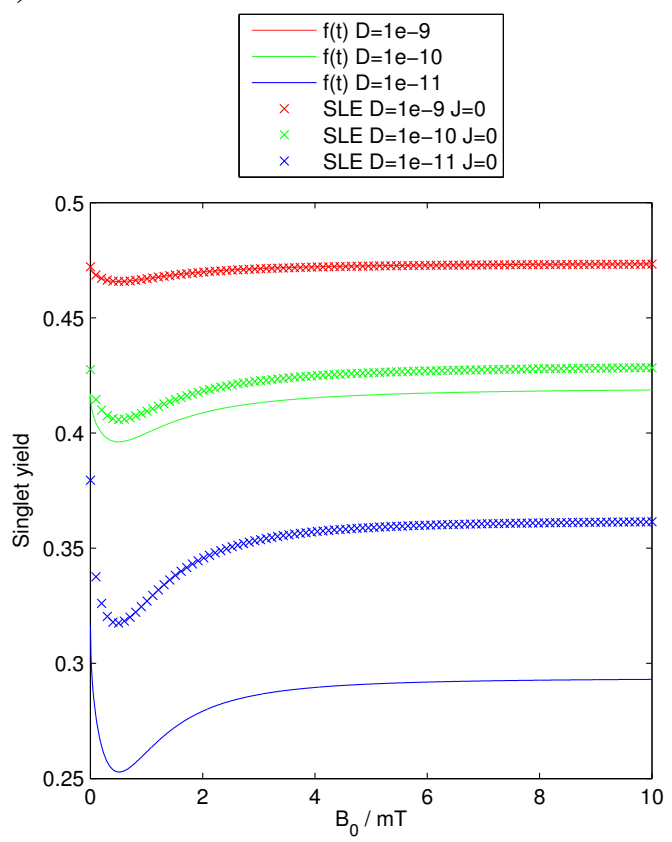

(C)

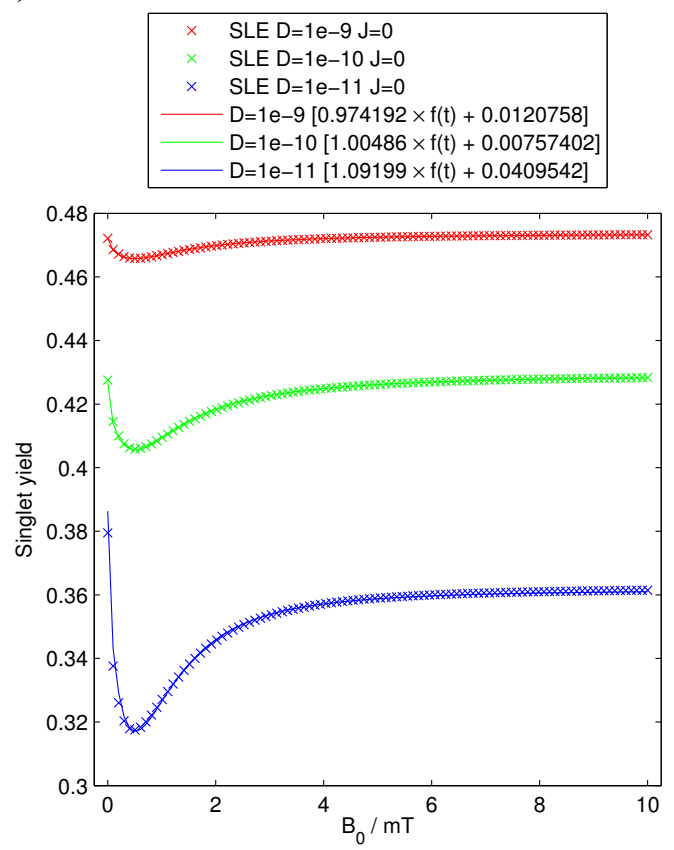

(B)

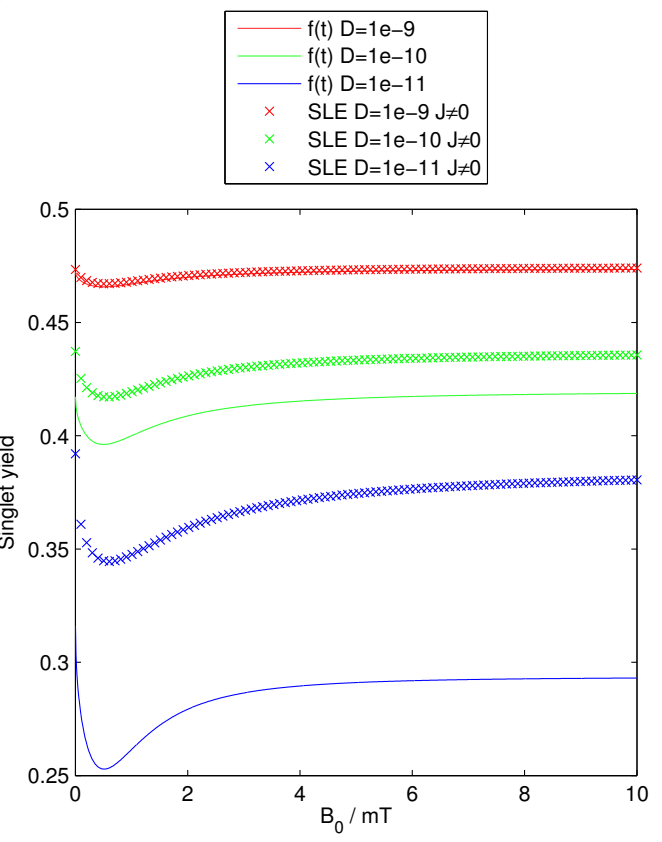

(D)

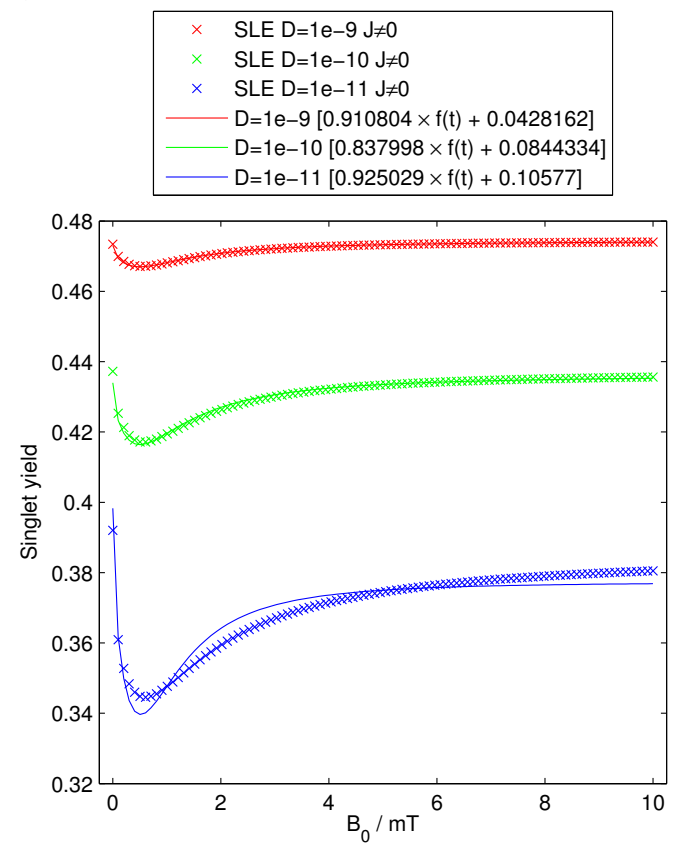

Figure S12. Effect of a magnetic field $(0 \leq B \leq 10 \mathrm{mT})$ on the singlet yield calculated using the stochastic Liouville equation (crosses) and the $f(t)$ method (lines). (A) \& (C): $J=0$. (B) \& (D): $J \neq$ 0. $D=10^{-9}$ (red), $10^{-10}$ (green), $10^{-11}$ (blue) $\mathrm{m}^{2} \mathrm{~s}^{-1}$. (C) \& (D) show the singlet yields calculated using the $f(t)$ method scaled and offset to match the SLE results. 


\section{References}

(1) Hansen, P. C., Rank-deficient and discrete ill-posed problems: numerical aspects of linear inversion. SIAM: 1998.

(2) Hansen, P. C. Regularization tools: a Matlab package for analysis and solution of discrete ill-posed problems.

(3) Brocklehurst, B. J. Chem. Soc. Faraday Trans. II 1976, 72, 1869-1884.

(4) Adrian, F. J., In Chemically Induced Magnetic Polarization, D. Reidel: 1977; Vol.

34, pp 77-105.

(5) Hansen, P. C. SIAM Review 1992, 34, 561-580.

(6) Bro, R.; de_Jong, S. J. Chemomet. 1997, 11, 393-401.

(7) Buck, B.; Macaulay, V. A., Maximum Entropy in Action. Oxford University Press: Oxford, 1991.

(8) Hore, P. J. J. Magn. Reson. 1985, 62, 561-567.

(9) Timmel, C. R.; Till, U.; Brocklehurst, B.; McLauchlan, K. A.; Hore, P. J. Mol. Phys. 1998, 95, 71-89.

(10) Pedersen, J. B.; Freed, J. H. J. Chem. Phys. 1973, 58, 2746-2762.

(11) Tsentalovich, Y. P.; Morozova, O. B.; Avdievich, N. I.; Ananchenko, G. S.;

Yurkovskaya, A. V.; Ball, J. D.; Forbes, M. D. E. J. Phys. Chem. A 1997, 101, 8809-8816.

(12) O'Dea, A. R.; Curtis, A. F.; Green, N. J. B.; Timmel, C. R.; Hore, P. J. J. Phys. Chem. A 2005, 109, 869-873. 